\title{
Antimatter Induced Fission for Nuclear Rocket Propulsion
}

\author{
T. Kammash* and R. Tang ${ }^{\dagger}$ \\ University of Michigan, Ann Arbor, MI, 48109
}

\begin{abstract}
A propulsion device in which antiprotons induced fission can be used to heat a suitable propellant to very high temperatures is presented. It is based on recent research which demonstrated that "at rest" annihilation of antiprotons in Uranium-238 nuclei induces fission at nearly $100 \%$ efficiency. The proposed device consists of an antiproton trap connected to a magnetic confinement system of the mirror configuration which will contain the pre-ionized propellant while being heated by the fission fragments and the annihilation products, namely the pions and muons. When an antiproton annihilates on a $\mathbf{U}^{238}$ nucleus, fission fragments with a total mass of $212 \mathrm{amu}$ and total energy of $160 \mathrm{MeV}$ are ejected nearly isotropically and interact almost instantly with the electrons of the propellant plasma transferring energy to them. The electrons subsequently transfer energy to the ions, and this energy exchange time dictates the plasma confinement time in the mirror. We use a $U^{238}$ propellant since it is effectively hazardless, and can provide sizable thrust because of its massive nucleus. When heated to one $\mathrm{keV}$ temperature by the fission fragments and the annihilation products, it is shown that such a system is capable of producing about 3000 seconds of specific impulse at a thrust of about 48 kilo-Newtons. When applied to a round trip mission to Mars, it is shown that it can be accomplished in about 127 days consisting of 2.2 days of thrusting and the rest in coasting. It is also shown the trip would require one gram of antiprotons.
\end{abstract}

\section{Nomenclature}

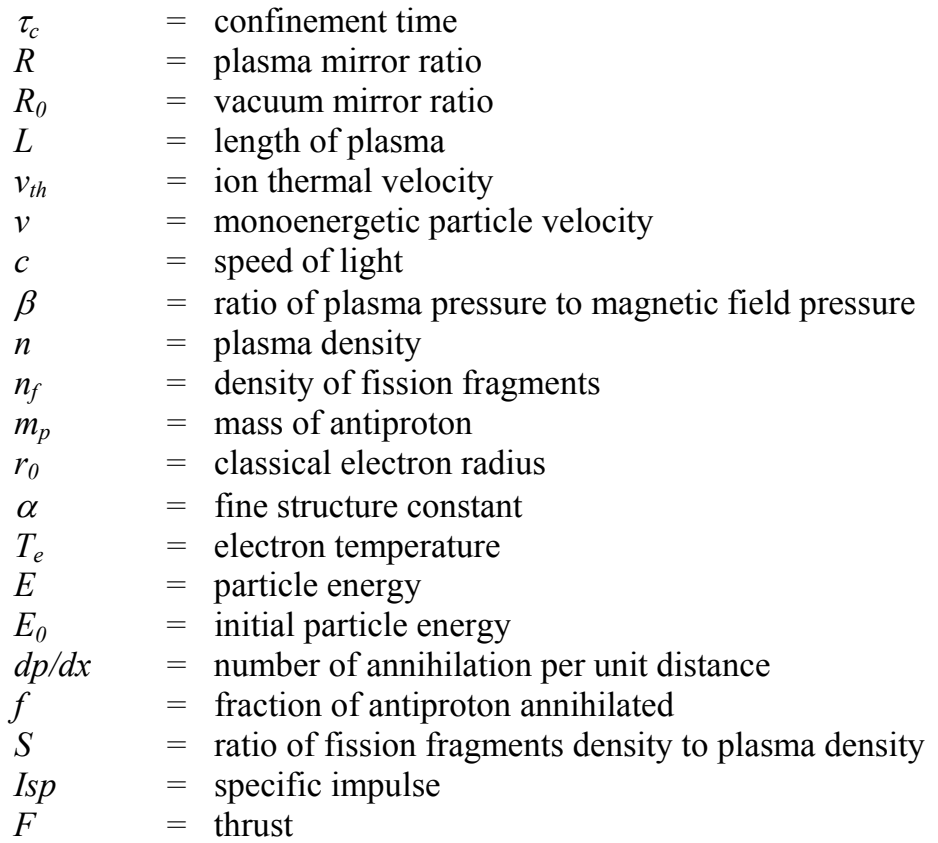

\footnotetext{
* Professor Emeritus, Dept. of Nuclear Engineering and Radiological Sciences, 2355 Bonisteel Blvd, Ann Arbor, MI 48109, AIAA Associate Fellow.

${ }^{\dagger}$ Graduate Student, Dept. of Aerospace Engineering, 1320 Beal Ave, Ann Arbor, MI 48109. 


\section{Introduction}

Several studies ${ }^{1,2}$ in recent years have shown that "at rest" annihilation of antiprotons in the Uranium isotope $\mathrm{U}^{238}$ Sleads to fission at nearly $100 \%$ efficiency. The resulting highly charged, fast fission fragments are highly ionizing, and can heat a suitable medium to very high temperatures. When an antiproton or a proton with multiple $\mathrm{MeV}$ kinetic energy slams into a target material it undergoes collisions with the electrons of the target and slows down by giving up energy to these particles. In the case of the proton it comes to rest in the material and forms a chemical bond with other atoms or diffuses around as atomic hydrogen. In the case of the antiproton it displaces an orbital electron around the nucleus, and begins immediately to cascade down in energy towards the ground state emitting x-rays as it makes these transitions. Eventually it enters the nucleus and an annihilation with either a neutron or a proton takes place. At this point the kinetic energy of the antiproton is measured in $\mathrm{eV}$, and not in $\mathrm{MeV}$, hence the label "at rest" annihilation. Nuclear fission following the annihilation at rest of antiprotons in heavy nuclei has been demonstrated in Uranium and Bismuth, and measurements have been made of the mass distribution of the fission fragments, as well as the multiplicity of the light charged particles that were emitted in the process. It was shown for example ${ }^{3}$ that in the case of uranium the average masses and kinetic energies of the fission fragments are respectively $212 \mathrm{amu}$ and $160 \mathrm{MeV}$. It was also shown that, on the average, the fission fragments left the target at an angle of $30^{\circ}$ with respect to the target plane, indicating that they emerged within a $60^{\circ}$ cone whose apex is at the target. We utilize this information to propose a propulsion device whose propellant is primarily "depleted" uranium, and where capabilities are such that human space exploration would be feasible in the near future.

\section{Concept Description and Analysis}

We envisage a propulsion system, illustrated in Fig. 1, which is based on the gasdynamic mirror ${ }^{4}$ magnetic configuration with asymmetric mirror ratios to allow the plasma confined in it to escape from one end to produce thrust. The plasma in question would be $\mathrm{U}^{238}$ singly ionized gas whose density and temperature are such that the ion-ion collision mean free path is much shorter than the length of the device. Under these conditions the plasma behaves likes a fluid and its escape is analogous to the flow of a gas into vacuum from a vessel with a hole. The confinement time $\tau_{c}$ of a plasma in the gasdynamic mirror (GDM) is given by

$$
\tau_{c}=\frac{R L}{v_{t h}}
$$

where $R$ is the plasma mirror ratio, $L$ the length, and $v_{t h}$ the ion thermal velocity. The plasma mirror ratio is related to the vacuum mirror ratio $R_{0}$ by

$$
R=\frac{R_{0}}{\sqrt{1-\beta}}
$$

where $\beta$ is the ratio of the plasma pressure to the magnetic field pressure. The principle of operation of such a propulsion device is to inject radially, at an appropriate axial position a $\mathrm{U}^{238}$ target, either in a solid strip form, or as an atomic beam form and strike it with an axially injected pulsed antiproton beam to induce fission. The nearly isotropically emitted fission fragments will slow down on the electrons of the $\mathrm{U}^{238}$ gas which is inserted in the GDM just prior to the injection of the beam to provide the background plasma (propellant). It also serves as the medium for the transportation of the antiprotons from the point of injection to the target. In a low temperature plasma of 6.5 $\mathrm{eV}$ (corresponding to the ionization energy of Uranium), an energetic antiproton can slow down on the electrons of the plasma or undergo annihilation reactions with the ions of the medium with the latter rate given by Eq. (3) (Ref. $5)$.

$$
\frac{d p}{d x}=0.19 n \pi r_{0}^{2}\left(\frac{\gamma}{1-e^{-\gamma}}\right) \frac{c}{v}
$$

where $n$ is the plasma density, $c$ the speed of light, $v$ the velocity of the antiproton, $r_{0}$ the classical electron radius $\left(2.82 \times 10^{-13} \mathrm{~cm}\right)$ and 


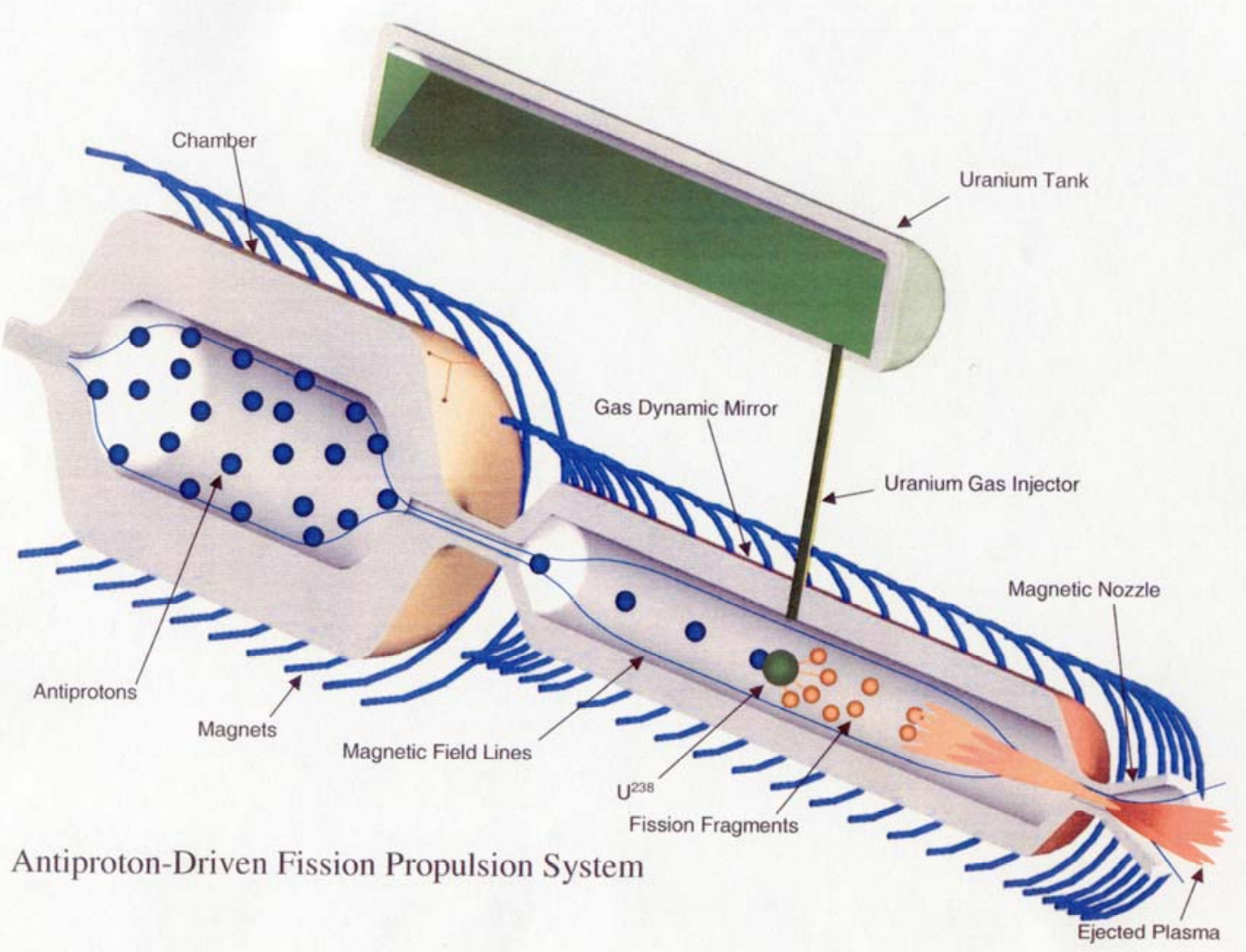

Figure 1. Schematics of the antiproton-driven fission propulsion system based on the GDM concept.

$$
\gamma=2 \pi \alpha \frac{c}{v}
$$

with $\alpha$ being the fine structure constant (1/137). The energy loss per unit time for the interaction with the electrons can be expressed by Eq. (5) (Ref. 6).

$$
\frac{d E}{d t}=v \frac{d E}{d x}=-c_{1} E
$$

where $E$ is the energy of the antiproton and $c_{1}$ is a constant given by

$$
c_{1}=2 \times 10^{-12} \frac{n}{m_{p} T_{e}^{3 / 2}(\mathrm{keV})}
$$

Here $m_{\mathrm{p}}$ represents the mass of the antiproton in atomic mass units (amu) and $T_{\mathrm{e}}$ the electron temperature in the plasma. The distance $x$ in the plasma which the antiproton must traverse from the injection point in order to reach a particular value of energy, $E$, is obtained by integrating Eq. (5) with the result

$$
E^{1 / 2}=E_{0}^{1 / 2}-c_{1} \sqrt{\frac{m_{p}}{8}} x
$$


where $E_{0}$ is the initial energy, namely that at $x=0$. If we further specify that the electron energy be $E_{\mathrm{c}}$ at a distance $x_{\mathrm{c}}$ then we can also write

$$
E(x)=\left[E_{c}^{1 / 2}+c_{1} \sqrt{\frac{m_{p}}{8}}\left(x_{c}-x\right)\right]^{2}
$$

The desired position for the annihilation is one that corresponds to a final energy of near zero so as to invoke the "at rest" condition alluded to earlier. The quantity of interest is $f$ which represents the fraction of antiprotons that annihilated after penetrating a given distance into the GDM device. It can be expressed in terms of $p$ as

$$
f=1-e^{-p}
$$

where $p(E)$ can be obtained from Eqs. (3) and (5), namely

$$
\frac{d p}{d E}=\frac{d p}{d x}\left(\frac{d E}{d x}\right)^{-1}
$$

The above equation can be integrated to yield

$$
p(E)=\frac{2 A}{k} \ln \left[\frac{e^{k E^{-1 / 2}}-1}{e^{k E_{0}^{-1 / 2}}-1}\right]
$$

where

$$
\begin{aligned}
& A=\frac{0.19 \pi^{2} r_{0}^{2} n \alpha c^{2}}{c_{1}} \sqrt{2 m_{p}} \\
& k=2 \pi \alpha c \sqrt{\frac{1}{2} m_{p}}
\end{aligned}
$$

Note that when $E=E_{0}, p=0$ and $f=0$ as expected, and that the dependence of $f$ on $x$ is through $p[E(x)]$ as may be seen from Eqs. (8) and (11). If we choose $n=10^{16} \mathrm{~cm}^{-3}$ to be the uranium plasma density in GDM whose temperature is $T_{\mathrm{e}}=6.5 \mathrm{eV}$ and assume that the antiprotons are injected at an energy of $20 \mathrm{keV}$, then they will have a final energy of $E_{\mathrm{c}} \approx 0$ at $\mathrm{x}_{\mathrm{c}}=27.8 \mathrm{~cm}$. This is the distance from the point of injection at which the $\mathrm{U}^{238}$ beam must be radially inserted in order to affect an "at rest" annihilation in the target. Upon initiating the fission in the $\mathrm{U}^{238}$ ions two fission fragments are produced per annihilation with a total mass of $212 \mathrm{amu}$ and total kinetic energy of $160 \mathrm{MeV}$. These fragments will heat the electrons of the Uranium plasma to a temperature given by Eq. (13) (Ref. 7).

$$
T_{e}=\frac{2}{3} \frac{S E_{0}}{1+S}
$$

where, in this case, $E_{0}$ is the energy of the fission fragment at birth, and

$$
S=\frac{n_{f}}{n}
$$

with $n_{f}$ denoting the density of the fission fragments. For the case of heating the electrons to $1 \mathrm{keV}$, the value of $S \approx$ $0.02 \times 10^{-3}$ and for a plasma density $n=10^{16}, n_{f}=2 \times 10^{11}$ which denotes the number of fission fragments per unit volume required to heat the electrons to $1 \mathrm{keV}$. These electrons will exchange energy with the ions of the plasma 
with a thermalization time of 0.012 seconds. This time dictates the confinement time in the pulsed GDM device which when substituted in Eqs. (1) and (2) along with the ion velocity corresponding to one keV yields for the length of the device $L=6.84 \mathrm{~m}$ having employed the following parameters: $\beta=0.95$ and $R=50$ which are consistent with stable plasma confinement. If we further specify a radius of the GDM chamber to be $50 \mathrm{~cm}$, then we find that the thrust generated by this propulsion device is 48 kilo-Newtons. The central magnetic field required to confine the plasma is found to be 2.06 tesla, and using the Virial theorem ${ }^{8}$ we find that the total mass of the magnets for the GDM and the antiproton trap to be about 8 metric tonnes. If we further add these masses to the structure and tankage masses and assume they constitute the total vehicle mass, the result is about 49 metric tonnes. Applying the propulsive parameters that characterize the system, namely $I s p=2.91 \times 10^{3} \mathrm{sec}$ and thrust $F=48 \mathrm{kN}$, to a round trip to Mars assuming certain periods of coasting in each of the out bound and return legs of the journey, we find that the complete journey takes 127 days. Of these, only 2.2 days of thrusting is required which in turn means about one gram of antiprotons is needed to accomplish the mission.

\section{Conclusion}

This paper has shown that antiproton annihilation in $\mathrm{U}^{238}$ nuclei is a source of energy that can be utilized in propulsion applications. The fission fragments that emerge from this reaction are sufficiently energetic to heat a uranium plasma to high enough temperatures that result in very desirable propulsive capabilities. When applied to a Mars mission, it is shown that such a journey can be accomplished in less than six months, requiring one gram of antiprotons.

\section{References}

${ }^{1}$ Hofmann, P., et al., "Fission of Heavy Nuclei Induced by Stopped Antiprotons. I. Inclusive Characteristics of Fission Fragments," Physical Review C, Vol. 49, No. 5, 1994, pp. 2555-2568.

${ }^{2}$ Kim, Y. S., et al., "Fission of Heavy Nuclei Induced by Stopped Antiprotons. II. Correlations between Fission Fragments," Physical Review C, Vol. 54, No. 5, 1996, pp. 2469-2476.

${ }^{3}$ Bocquet, J. P., et al., "Prompt Fission Induced by Antiproton Annihilation at Rest on Heavy Nuclei," Z. Phys. A-Hadrons and Nuclei, Vol. 342, No. 2, 1992, pp. 183-189.

${ }^{4}$ Kammash, T., and Lee, M. J., "Gasdynamic Fusion Propulsion System for Space Exploration," Journal of Propulsion and Power, Vol. 11, No. 3, 1995, pp. 544-553.

${ }^{5}$ Morgan, D. L., Jr., "Annihilation Localization in Gas-Core and Plasma-Core Annihilation Rocket Engines," Acta Astronautica, Vol. 21, No. 1, 1990, pp. 23-27.

${ }^{6}$ Kammash, T., Fusion Reactor Physics - Principles and Technology, Ann Arbor Science Publishers, Ann Arbor, MI, 1975 , pp. 81-129.

${ }^{7}$ Kammash, T., and Galbraith, D. L., “Antimatter-Driven Fusion Propulsion Scheme for Solar System Exploration,” Journal of Propulsion and Power, Vol. 8, No. 3, 1992, pp. 644-649.

${ }^{8}$ Litchford, R. J., Bitteker, L. J., and Jones, J. E., "Prospects for Nuclear Electric Propulsion Using Closed-Cycle Magnetohydrodynamic Energy Conversion,” NASA/TP-2001-211274, Marshall Space Flight Center Report (2001). 